\title{
Headache related to mask use of healthcare workers in COVID-19 pandemic
}

\author{
Cansu Köseoğlu Toksoy ${ }^{1}$, Hayri Demirbaş ${ }^{1}$, Erhan Bozkurt ${ }^{2}$, Hakan Acar ${ }^{1}$, and Ülkü Türk Börü ${ }^{1}$ \\ 'Department of Neurology, Afyonkarahisar Health Sciences University School of Medicine, Afyonkarahisar, Turkey \\ ${ }^{2}$ Department of Internal Medicine, Afyonkarahisar Health Sciences University School of Medicine, Afyonkarahisar, Turkey
}

Received December 15, 2020

Revised February 2, 2021

Accepted February 16, 2021

Handling Editor: Jiseok Baik

\section{Correspondence}

Cansu Köseoğlu Toksoy

Department of Neurology, Afyonkarahisar

Health Sciences University School

of Medicine, Zafer Health Complex

Dörtyol District 2078 Street No: 3,

Afyonkarahisar 03030, Turkey

Tel: +90-532-732-46-24

Fax: +90-272-228-14-29

E-mail: dr.cansukoseoglu@gmail.com
Background: It has been reported that a new type of headache may develop as a result of face mask use during the COVID-19 pandemic. The aim of this study is to investigate the clinical features of face mask-related headache during the COVID-19 pandemic.

Methods: This is a cross-sectional study carried out on healthcare workers at the Afyonkarahisar Health Sciences University. The number of workers at our university was established. Sample size was calculated using the G*Power program. A questionnaire consisting of questions relating to pre-existing headache, an aggravation in headache, and de-novo headache was filled out by 3 neurologists with all participants.

Results: Data was collected from a total of 375 participants, after the exclusion of 5 individuals who refused to participate. Out of all participants, 26 (6.9\%) used a filtering mask, 274 (73.1\%) used a surgical mask, 75 (20.0\%) participants used a combination of both masks. The number of participants with preexisting headache was 114 (30.4\%) had pre-existing headache. Of those with pre-existing headache, 77 (67.5\%) healthcare workers had reported an aggravation in their headache after mask use. De-novo headache was observed in 116 (30.9\%) of participants. De-novo headache characteristics included throbbing in 17 (14.7\%) participants and pressing in 99 (85.3\%) participants. In addition, symptoms such as tachypnea, sleep disturbance, and fatigue were found to be significantly higher.

Conclusions: This study indicates that healthcare workers develop headaches due to use of masks during the COVID-19 outbreak.

Key Words: COVID-19; Eyeglasses; Fatigue; Headache; Health Personnel; Masks; Pandemics; Personal Protective Equipment; Sleep Wake Disorders; Tension-Type Headache.

\section{INTRODUCTION}

COVID-19, which started in Wuhan, China in December and spread rapidly around the world, was declared a pandemic by the World Health Organization in March 2020 and has been observed in our country since March
2020. Since the disease is transmitted through respiratory droplets, it is necessary to use masks and personal protective equipment. In particular, the long-term exposure of healthcare workers to masks and personal protective equipment can cause many complaints such as acne, skin breakdown, impaired cognition, and headache [1]. There (c) This is an open-access article distributed under the terms of the Creative Commons Attribution Non-Commercial License (http://creativecommons.org/licenses/by-nc/4.0/), which permits unrestricted non-commercial use, distribution, and reproduction in any medium, provided the original work is properly cited.

(C) The Korean Pain Society, 2021
Author contributions: Cansu Köseoğlu Toksoy: Writing/manuscript preparation; Hayri Demirbaş: Data collection; Erhan Bozkurt: Methodology; Hakan Acar: Data collection; Ülkü Türk Börü: Supervision. 
are several studies showing the emergence of a new headaches due to the use of masks in healthcare workers [2-4]. The aim of this study is to investigate mask-related headache during the COVID-19 outbreak, and to determine its clinical features.

\section{MATERIALS AND METHODS}

This study was carried out at the Afyonkarahisar Health Sciences University between November 15 and December 15,2020 . It was performed on doctors, nurses, and other healthcare professionals working at the hospital.

\section{Sample size calculation}

There were 1,734 healthcare workers at our hospital. Three hundred fifty-one of them were doctors, 462 were nurses, and 921 were other healthcare professionals. The G*Power package program (Heinrich-Heine-Universität Düsseldorf, Düsseldorf, Germany) was used for calculations. The minimum sample size was calculated as 310 , considering the effect size as medium (0.3), the type I error rate $=0.05$ and power $=95 \%$.

\section{Screening form}

A questionnaire form consisting of 30 parameters was prepared. Age, sex, department of work, duty, chronic diseases, type of mask used, daily mask use duration, use of other protective equipment, presence of pre-existing headache, characteristics of previous headache, whether headache was aggravated after mask use, whether there was an increase in analgesic drug use, whether a new headache appeared, the characteristics of the new headache, how long after wearing a mask the headache started, how long after removing the mask the headache stopped, and any accompanying symptoms were all recorded. All participants were questioned face to face by 3 neurologists and the questionnaire was filled out.

\section{Ethical approval}

This study was approved by the Local Ethics Committee (Protocol No: 2020/448). The study was conducted in accordance with the principles of the Declaration of Helsinki.

\section{Statistical analysis}

Descriptive analysis was used to study baseline characteristics. The Pearson-chi-square test was used for the evaluation of categorical data and the Mann-Whitney test was used for the evaluation of quantitative data. Whether or not the quantitative data had normal distribution was evaluated using the Kolmogorov-Smirnov and ShapiroWilk tests. The level of significance was chosen as 0.05 . All statistical analyses were performed using the SPSS 20.1 package program version for Windows (IBM Co., Armonk, NY).

\section{RESULTS}

A total of 380 healthcare professionals were invited to participate in this study, and 375 accepted, for a participation rate of $98.7 \%$. One hundred sixty-one (42.9\%) of the participants were male, and 214 (57.1\%) of them were female. The median age of the study participants was 32 (interquartile range [IQR]: 12, range 21-65). Seventy-eight (20.8\%) of the participants were doctors, $103(27.5 \%)$ were nurses, and 194 (51.7\%) were other healthcare workers. One hundred five $(28.0 \%)$ of them were working in the COVID clinic and COVID intensive care unit (ICU), while $270(72.0 \%)$ were working in the polyclinic and other departments. Comorbidity was present in $29(7.7 \%)$ of all screened healthcare workers. Table 1 summarizes the characteristics of the healthcare workers.

Twenty-six (6.9\%) person used filtering masks, 274

Table 1. Characteristics of healthcare workers

\begin{tabular}{lc}
\hline Characteristic & Healthcare workers \\
\hline Sex & $214(57.1)$ \\
Female & $161(42.9)$ \\
Male & \\
Occupation & $78(20.8)$ \\
Doctor & $103(27.5)$ \\
Nurse & $194(51.7)$ \\
Other healthcare workers & \\
Department & $105(28.0)$ \\
COVID clinic and ICU & $270(72.0)$ \\
Other departments & $29(7.7)$ \\
Comorbidities & $6(1.6)$ \\
Hypertension & $5(1.3)$ \\
Diabetes mellitus & $5(1.3)$ \\
Asthma & $5(1.3)$ \\
Hypothyroidism & $1(0.3)$ \\
Hypertiroidism & $1(0.3)$ \\
Atrial fibrillation & $2(0.5)$ \\
Epilepsy & $3(0.8)$ \\
Hyperlipidemia & $1(0.3)$ \\
Multiple sclerosis &
\end{tabular}

Values are presented as number (\%).

ICU: intensive care unit. 
Table 2. Mask usage

\begin{tabular}{cc}
\hline Characteristic & Healthcare workers \\
\hline Mask type & $26(6.9)$ \\
Filtering mask & $274(73.1)$ \\
Surgical mask & $75(20.0)$ \\
Filtering + surgical mask & \\
Mask usage duration (hr) & $29(7.7)$ \\
$1-4$ & $346(92.3)$ \\
$>4$ & \\
\hline
\end{tabular}

Values are presented as number (\%).

(73.1\%) used surgical masks, and 75 (20.0\%) used both masks in combination. Mask use duration was between 1-4 hours in 29 healthcare workers and more than 4 hours in 346 healthcare workers (Table 2). One hundred four (27.7\%) of them were using other protective equipment including eyeglasses. Seventy-eight (20.8\%) were using other protective equipment other than eyeglasses. One hundred ninety-three $(51.5 \%)$ of them were not using other protective equipment.

Pre-existing headache was recorded in 114 (30.4\%) healthcare workers. Seventy-nine of them were female and 35 were male. Pre-existing headache in female was statistically significantly higher $(P=0.002)$. Pre-existing headache type was migraine in $43(37.7 \%)$, tension headache in $68(59.6 \%)$, and other headaches in 3 healthcare workers (2.6\%) (Table 3). The pre-existing headache median frequency was 3 (IQR: 3 ), and the visual analogue scale (VAS) score was 5 (IQR: 3 ). Seventy-seven (67.5\%) of those with pre-existing headache had an aggravation in headache after mask use. There was an aggravation in headache in $7.7 \%$ in those who used filtering masks, in $19 \%$ of those using surgical masks, and in $30.7 \%$ of those who used both in combination. There was a statistically significant aggravation in headache in those who used both masks in combination $(P=0.021)$. There was no significant correlation between the duration of mask use and the aggravation in headache $(P=0.350)$. There was no significant correlation between the department and duty of the individuals and the aggravation in headache $(P=0.067$ and $P=0.133$, respectively). There was an aggravation in headache in 27 (26.0\%) of those using other protective equipment including eyeglasses, in $15(19.2 \%)$ of those using other protective equipment except eyeglasses, and in $32(16.6 \%)$ of those who were not using other protective equipment. There was no significant relationship between the use of other protective equipment including eyeglasses and aggravation in headache $(P=0.133)$. The median frequency of those with aggravated headache was 6 (IQR: 12), and the VAS score was 6 (IQR: 7). There was a statistically significant increase in frequency and VAS scores $(P<0.001$ and $P=0.019$, re-
Table 3. Pre-existing headache characteristics

\begin{tabular}{cc}
\hline \multicolumn{1}{c}{ Condition } & Healthcare workers \\
\hline Sex & \\
Female & $79(69.2)$ \\
Male & $35(30.7)$ \\
Pre-existing headache diagnosis & \\
Migraine & $43(37.7)$ \\
Tension-type headache & $68(59.6)$ \\
Other & $3(2.6)$ \\
\hline
\end{tabular}

Values are presented as number (\%).

spectively). There was an increase in analgesic drug use in 55 healthcare workers (48.2\%). No significant relationship was observed between type of mask and the duration of mask use and analgesic drug increase $(P=0.069$ and $P=$ 0.075 , respectively).

De-novo headache was recorded in 116 (30.9\%) healthcare workers. De-novo headaches were detected in $50.0 \%$ of those who used filtering masks, in $25.9 \%$ of those using surgical masks, and in $42.7 \%$ of those who used both in combination. A significant relationship was found between the type of mask and new headache $(P=0.002)$. No relationship was found between mask use duration and de-novo headache $(P=0.097)$. De-novo headache was recorded in 42 (40.4\%) of those using other protective equipment including eyeglasses, in $22(28.2 \%)$ of those using other protective equipment except eyeglasses, and in 52 (26.9) of those who were not using other protective equipment. A significant relationship was found between the use of other protective equipment including eyeglasses and de-novo headache $(P=0.048)$. De-novo headache was observed in $41.9 \%$ of COVID clinic and ICU workers, and in $26.7 \%$ of workers in other departments. A significant relationship was found between the department of employment and de-novo headache $(P=0.004)$. There was no significant relationship between task and de-novo headache $(P=0142)$. De-novo headache characteristics were throbbing in $17(14.7 \%)$ healthcare workers, pressing in 99 (85.3\%), unilateral in 12 (10.3\%), and bilateral in 104 (89.7\%) (Table 4). The median frequency of de-novo headache was 8 (IQR: 7), and the VAS score was 5 (IQR: 2). De-novo headache areas are shown in Fig. 1. Accompanying symptoms are sleep disorder, fatigue, tachypnea, dizziness, nausea, and palpitations (Table 4). The median value of the onset of headache after wearing a mask was 1 (IQR: 2), and the median value of headache resolution time after mask removal was 2 (IQR: 3). 


\section{DISCUSSION}

The results of this study indicate that during the COVID-19 outbreak, a new type of headache has developed among healthcare workers. Both an aggravation in pre-existing headaches and the emergence of de-novo headaches has been shown to increase with mask use, regardless of mask use duration. This rate increases even more when both masks are used in combination. A de-novo headache is characterized by a bilateral, moderate, and pressing nature. The clinical characteristics of a de-novo headache are more like a tension headache, however, it has also some accompanying symptoms, such as throbbing and unilateral characteristics similar to migraines. The new type headache starts shortly after putting on the mask and disappears after removing it. In addition, sleep disorder, fatigue, and tachypnea were also observed at a high rate.

Rosner [1] reported the adverse effects of long-term mask use on healthcare workers, and headache was the most common complaint observed, at a rate of $71.4 \%$.

The number of studies on headache associated with

Table 4. De-novo headache characteristics

\begin{tabular}{lr}
\hline \multicolumn{1}{c}{ Characteristic } & Value \\
\hline Throbbing & $17(14.7)$ \\
Pressing & $99(85.3)$ \\
Unilateral & $12(10.3)$ \\
Bilateral & $104(89.7)$ \\
Time between wearing mask to onset of headache & 1 (IQR 2) \\
$\quad$ (median hr) & \\
Time from removal of mask to resolution of headache & $2($ IQR 3) \\
$\quad$ (median hr) & \\
Associated symptoms & \\
$\quad$ Nausea & $42(11.2)$ \\
$\quad$ Dizziness & $65(17.3)$ \\
$\quad$ Sleep disorder & $94(25.1)$ \\
$\quad$ Tachypnea & $78(20.8)$ \\
$\quad$ Palpitation & $11(2.9)$ \\
\hline
\end{tabular}

Values are presented as number (\%).

IQR: interquartile range. mask use is very limited [2-4]. Several studies have described mask-associated de-novo headache $[2,3]$. It was reported that a significant increase was observed in headaches in people with pre-existing headache as well as an increase in analgesic drug use and a significant relationship was found between the use of other protective equipment, including goggles, and de-novo headache [2]. An another study reported that an increase was observed in preexisting headache and work stress after mask use. In the same study, work stress was found to be higher in patients with de-novo headache than those without headache [3]. In our study, the effect of work stress on headache could not be determined because an evaluation was not made regarding work stress.

There may be more than one mechanism in the physiopathology of de-novo headache. Several factors may explain its association with the use of filtering masks, including hypoxia, hypercapnia, local compression, and even mask use anxiety [4]. Sleep deprivation, irregular mealtimes, and emotional stress are other sources of headache among healthcare workers during prolonged mask use [2].

Tight-fitting masks cause insufficient ventilation, and cause the level of carbon dioxide $\left(\mathrm{CO}_{2}\right)$ to rise. $\mathrm{CO}_{2}$ is known as a respiratory stimulant, and inhalation of $\mathrm{CO}_{2}$ between the mask and the face causes increased respiratory activity [5]. In our study, the significantly higher incidence of de-novo headache in filtering mask users supports that $\mathrm{CO}_{2}$ inhalation may be a reasonable etiology. The fact that the highest aggravation in pre-existing headache was found in those who use a filter mask and surgical mask together supports this theory; but it is higher in those who use only surgical masks than in those who use only filtered masks, suggesting that there may be other factors besides $\mathrm{CO}_{2}$ inhalation. In a study of 130 astronauts exposed to high $\mathrm{CO}_{2}$ pressures during controlled training, the exposed group showed a significantly higher incidence of headache, in addition to respiratory symptoms and difficulty concentrating [6]. Another mechanism that can explain headache physiopathology may be external compression [7]. In our study, de-novo headache was more common in those who wear eyeglasses.

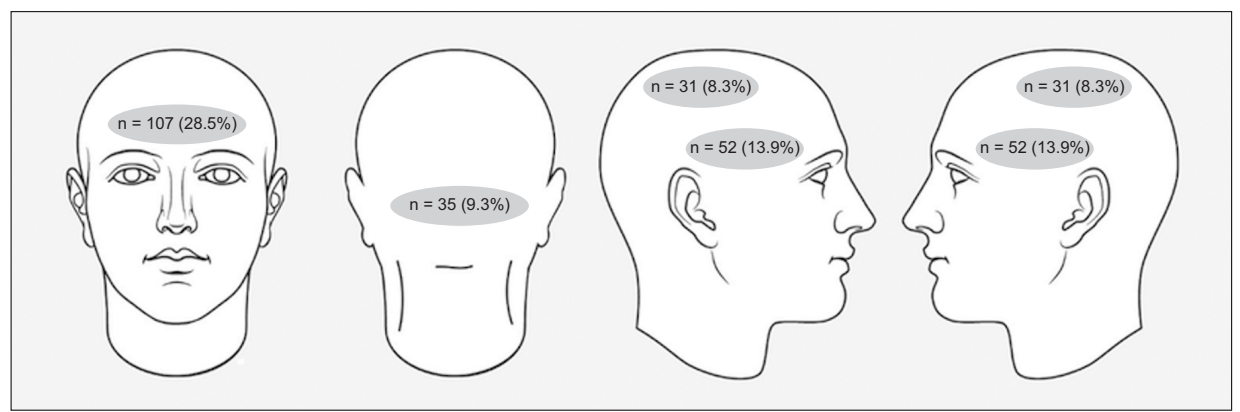

Korean J Pain 2021;34(2):241-245
Fig. 1. De-novo headache localizations. 
The limitations of this study are that other predisposing factors that may contribute to the development of de-novo headaches such as stress, nutrition, and sleep disorders were not evaluated.

The strengths of this study are that the study was conducted by neurologists who filled out the questionnaires face to face with the participants.

In conclusion, this study illustrates that mask use among healthcare workers during the COVID-19 outbreak causes de-novo headaches. The characteristics of the de-novo headache are similar to tension-type headache. Better strategies are needed to reduce the duration of exposure to personal protective equipment so that healthcare workers' job performance and quality of life are less affected. Shorter duty shifts and correspondingly shorter durations of personal protective equipment use may well be a good strategy to avoid the negative effects of using such equipment.

\section{CONFLICT OF INTEREST}

No potential conflict of interest relevant to this article was reported.

\section{FUNDING}

No funding to declare.

\section{ORCID}

Cansu Köseoğlu Toksoy, https://orcid.org/0000-0002-9224-9203
Hayri Demirbaș, https://orcid.org/0000-0002-8339-7765

Erhan Bozkurt, https://orcid.org/0000-0002-1853-7098

Hakan Acar, https://orcid.org/0000-0002-3429-2318

Ülkü Türk Börü, https://orcid.org/0000-0002-0094-5624

\section{REFERENCES}

1. Rosner E. Adverse effects of prolonged mask use among healthcare professionals during COVID-19. J Infect Dis Epidemiol 2020; 6: 130.

2. Ong JJY, Bharatendu C, Goh Y, Tang JZY, Sooi KWX, Tan YL, et al. Headaches associated with personal protective equipment - a cross-sectional study among frontline healthcare workers during COVID-19. Headache 2020; 60: 864-77.

3. Ramirez-Moreno JM, Ceberino D, González-Plata A, Rebollo B, Macías-Sedas P, Ramchandani RH, et al. Mask-associated de novo headache in healthcare workers during the COVID-19 pandemic. medRxiv. 2020.08.07.20167957 [Preprint]. 2020 [cited 2020 Dec 30]. Available from: https://doi.org/10.11 01/2020.08.07.20167957.

4. Lim EC, Seet RC, Lee KH, Wilder-Smith EP, Chuah BY, Ong BK. Headaches and the N95 face-mask amongst healthcare providers. Acta Neurol Scand 2006; 113: 199-202.

5. Johnson AT. Respirator masks protect health but impact performance: a review. J Biol Eng 2016; 10: 4.

6. Law J, Young M, Alexander D, Mason SS, Wear ML, Méndez $\mathrm{CM}$, et al. Carbon dioxide physiological training at NASA. Aerosp Med Hum Perform 2017; 88: 897-902.

7. Krymchantowski AV. Headaches due to external compression. Curr Pain Headache Rep 2010; 14: 321-4. 